\title{
ОТРАСЛЕВАЯ ЭКОНОМИКА
}

УДК 658.14/.17:336.531.2(470+571), ББК У29

(C) Кормишкина Л.А., Юленкова И.Б.

\section{Финансирование инвестиционного процесса в российском бизнесе: оценка, тенденции, проблемы*}

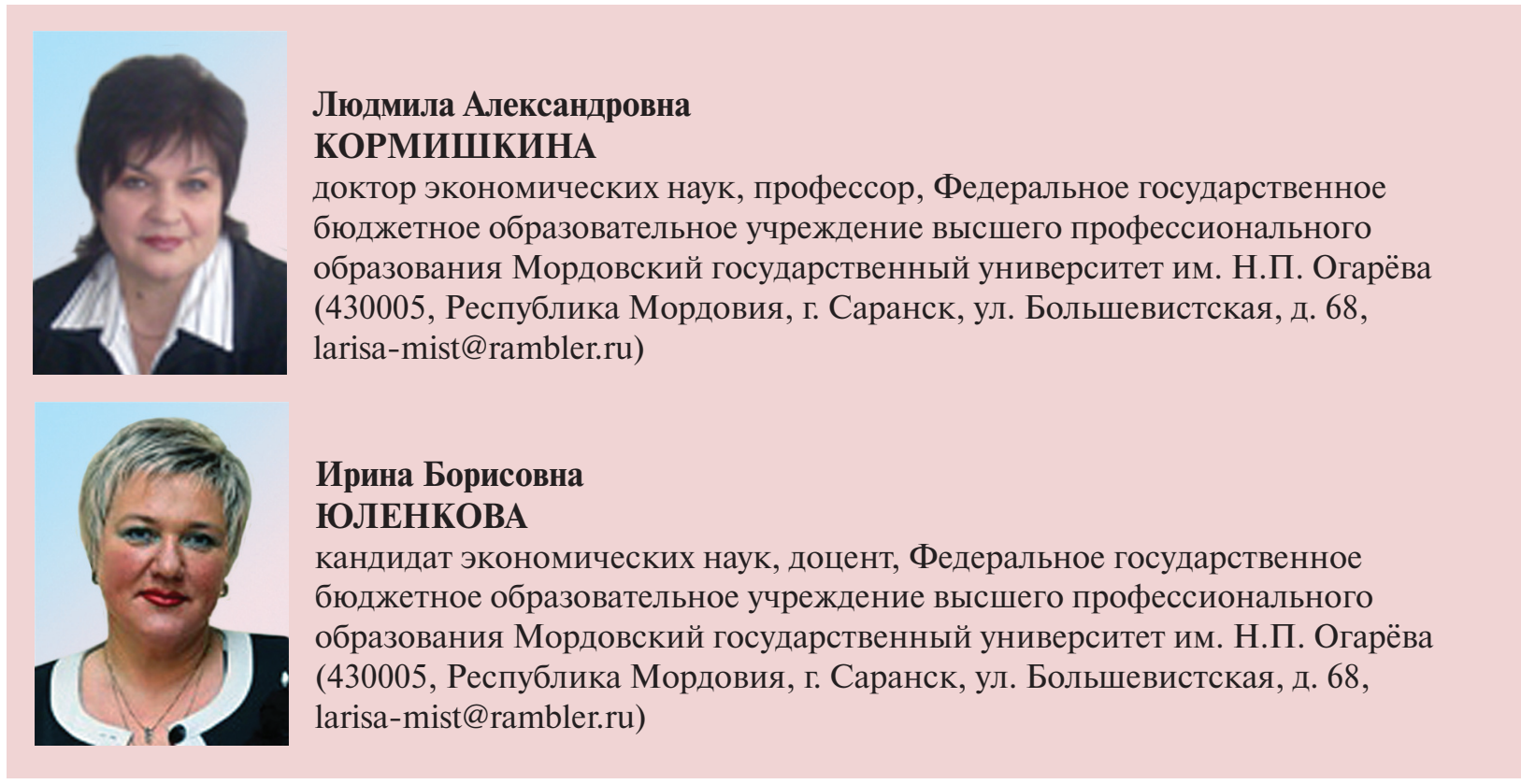

Аннотация. В современных условиях обеспечение финансирования инвестиционного процесса становится важнейшей задачей государственной экономической политики, направленной на достижение устойчивого роста и динамичного развития российской экономики в целом. Решение этой проблемы требует её дальнейшего теоретического осмысления, а также разработки соответствующего методологического обеспечения, методических и практических рекомендаций. Финансирование развития инвестиционной деятельности должно базироваться на использовании системного подхода, который позволяет рассматривать данный процесс как элемент системы финансовой поддержки инновационного развития государства. Необходимы научные исследования с целью расширения финансовой составляющей такой поддержки, создания стимулов для развития инвестиционного процесса, улучшения финансовых отношений в сфере формирования и использования объектов интеллектуальной собственности, выработки комплекса мер по изучению возможностей реализации нововведений на практике. Несмотря на то что различным аспектам рассматриваемой проблемы в экономической науке уделено достаточно много внимания,

* Статья подготовлена при финансовой поддержке РГНФ (проект № 134-02-00046а «Развитие теории и методологии исследования и обеспечения посткризисного экономического роста в контексте парадигмы неоиндустриального развития»). 
в настоящее время отсутствуют исследовательские работы, посвященные изучению источников финансирования инвестиционного процесса. Требуется разработка методических и практических рекомендаций по созданию в стране системы его финансирования с учетом современных мировых экономических тенденций. Авторами определено общеэкономическое значение прибыли предприятий в обеспечении финансовыми ресурсами устойчивого экономического роста. Теснота взаимосвязи рассчитывается на основе регрессионной модели, характеризующей отраслевое распределение валовой прибыли. Корреляционно-регрессионный анализ в рамках статьи позволил дать оценку степени влияния на индекс инвестиций в основной капитал в Российской Федерации основных источников его формирования, которые в силу своей экономической природы определяют его величину и динамику.

Ключевые слова: инвестиционный процесс, инвестиционный климат, бизнес, активизация, прибыль, источник финансирования, инвестиционные затраты, регрессионная модель, индексы, динамика, инвестиционная деятельность, инвестиционная активность, уровень налогообложения.

Инвестиционный климат каждой страны формируется при активном участии государства. Оно определяет внешнюю и внутреннюю политику; вводит налоги; устанавливает процентные ставки, меняя их в зависимости от экономической ситуации и сдерживая их рост специальными экономическими методами; воздействует на уровень собираемости налогов; активно занимается разработкой законодательных актов, формируя тем самым экономические, политические, правовые условия, определяющие функционирование предприятий.

«Изучение теоретико-методологических основ обеспечения устойчивого экономического роста позволяет заключить, что главную роль в решении данной задачи играют инвестиции» [1, с. 48]. Под воздействием функционирующего в экономике России так называемого механизма гашения инвестиционной активности в 1999-2007 гг. спад общественного производства сменился более тяжелой формой экономического кризиса в виде спада воспроизводства [2, с. 38-39]. При спаде воспроизводства ввод активной части основных фондов либо намного отстает от их выбытия, либо не компенсирует последнего. К основным причинам сло- жившейся ситуации можно отнести $48 \%$ износ основных фондов [3, с. 345]. Следует помнить, что обеспечение экономического развития возможно лишь за счет обеспечения расширенного воспроизводства, что предполагает возобновление основных элементов производительных сил во все увеличивающихся масштабах и их качественное совершенствование.

Казалось бы, с 2000 г. в отечественной экономике удалось преодолеть негативную тенденцию опережения выбытия активной части основных фондов над их вводом. Однако объемы вводимого оборудования остаются явно недостаточными, если исходить из индикаторов экономической безопасности и народно-хозяйственной задачи модернизации производственных мощностей и рабочих мест. Для сравнения: в 1970 году, определяемом как «эпоха застоя», коэффициент обновления составил $10,2 \%$, а выбытия $-1,7 \%$, т.е. обновление основных фондов шло в несколько раз быстрее, чем в 2011 году, когда коэффициенты их обновления и выбытия составили соответственно 4,6 и 0,8\% [3, с. 345]. Естественно, что при такой динамике обновления основных фондов сохраняется тенденция их высокого физического и морального износа. 
«Не способствует решению задачи инвестирования модернизации экономики и бюджетно-финансовая политика российского государства. Система государственного регулирования в этой сфере нуждается в существенной корректировке. Как показывают исследования ИСЭРТ РАН, повышение собираемости налогов и ряд непопулярных мер, прежде всего в среде крупного бизнеса, позволили бы привлекать в бюджетную систему страны 8-13 трлн. руб. в год. Однако практических шагов в этом направлении пока не сделано. Кроме того, в настоящее время не участвуют в решении приоритетных задач социально-экономического развития и мощные финансовые активы в виде средств резервного фонда, национального фонда благосостояния» $[1$, с. 56].

Подобная ситуация далеко не безобидна для национальной экономики, поскольку существенно снижается способность к инновационному развитию. Действие механизма гашения инвестиционной активности в производстве и экономического роста в современной экономике России означает, что само национальное хозяйство не может преодолеть сложившуюся ситуацию и обеспечить устойчивое экономическое развитие. Для этого требуются целый комплекс мер государственного регулирования и огромные затраты, способные прежде всего активизировать инвестиционную деятельность. В противном случае остается надеяться только на привлечение иностранного капитала, что в конечном счете приводит к возникновению угрозы экономической безопасности страны.

В результате приходится констатировать, что в современной российской экономике все еще присутствуют проявления многостороннего (общего) финансового кризиса. Его основные формы: убыточность, разрушение основного капитала, неплатежеспособность, криминализация финансового менеджмента, сознательное сокрытие финансовых потоков от учета, контроля, налогообложения, хищения финансовых ресурсов и др. Необходимо подчеркнуть, что формирование налоговой базы, способность предприятий к уплате налогов неразрывно связаны с финансовым состоянием последних.

Не вызывает сомнения справедливость вывода ученых и практиков о том, что высокий уровень налогообложения в России крайне негативно сказывается на инвестиционной деятельности.

В условиях современной рыночной экономики активизация инвестиционной деятельности предприятий прежде всего связана с поиском источников и видов финансовых ресурсов, которые должны обеспечить баланс между инвестиционными затратами и финансовыми возможностями.

Как известно, в результате приватизации главным источником финансирования инвестиционной деятельности являлись собственные средства организаций, на долю которых в РФ в 2011 г. приходилось почти $70 \%$ всего объема инвестиций. Эти средства формируются преимущественно за счет прибыли и амортизационных отчислений организации (табл. 1).

Прибыль является основным внутренним источником формирования финансовых ресурсов предприятия, обеспечивающих его развитие. Чем выше уровень генерирования прибыли в процессе хозяйственной деятельности, тем меньше потребность в привлечении финансовых средств из внешних источников и, при прочих равных условиях, тем выше уровень самофинансирования его развития и конкурентные позиции на рынке. При этом, в отличие от некоторых других внутренних источников формирования финансовых ресурсов предприятия, при- 
Финансирование инвестиционного процесса в российском бизнесе: оценка, тенденции, проблемы

Таблица 1. Источники финансирования в основной капитал в российской экономике, \% к итогу

\begin{tabular}{|c|c|c|c|c|c|c|c|c|c|c|c|c|}
\hline \multirow{2}{*}{ Показатель } & \multicolumn{12}{|c|}{ Год } \\
\hline & 2000 & 2001 & 2002 & 2003 & 2004 & 2005 & 2006 & 2007 & 2008 & 2009 & 2010 & 2011 \\
\hline $\begin{array}{l}\text { Инвестиции в основной } \\
\text { капитал }\end{array}$ & 100 & 100 & 100 & 100 & 100 & 100 & 100 & 100 & 100 & 100 & 100 & 100 \\
\hline $\begin{array}{l}\text { В том числе по источникам } \\
\text { фрнансирования: } \\
\text { собственные средства }\end{array}$ & 47,5 & 49,4 & 45,0 & 45,2 & 45,4 & 44,5 & 42,1 & 40,4 & 39,5 & 37,1 & 41,0 & 42,7 \\
\hline $\begin{array}{l}\text { Из них: } \\
\text { - прибыль, остающаяся в } \\
\text { распоряжении организации } \\
\text { (фонд накопления) }\end{array}$ & 23,4 & 24,0 & 19,1 & 17,8 & 19,2 & 20,3 & 19,9 & 19,4 & 18,4 & 16,0 & 17,1 & 17,2 \\
\hline - амортизация & 18,1 & 18,5 & 21,9 & 24,2 & 22,8 & 20,9 & 19,2 & 17,6 & 17,3 & 18,2 & 20,5 & 21,6 \\
\hline - привлеченные средства & 52,5 & 50,6 & 55,0 & 54,8 & 54,6 & 55,5 & 57,9 & 59,6 & 60,5 & 62,9 & 59,0 & 57,3 \\
\hline $\begin{array}{l}\text { Из них: } \\
\text { - кредиты банков }\end{array}$ & 2,9 & 4,4 & 5,9 & 6,4 & 7,9 & 8,1 & 9,5 & 10,4 & 11,8 & 10,3 & 9,0 & 7,7 \\
\hline - бюджетные средства & 22,0 & 20,4 & 19,9 & 19,6 & 17,8 & 20,4 & 20,2 & 21,5 & 20,9 & 21,9 & 19,5 & 18,8 \\
\hline
\end{tabular}

быль является постоянно воспроизводимым источником и ее воспроизводство в условиях успешного хозяйствования осуществляется на расширенной основе.

Из таблицы 1 видно, что собственные средства предприятий в инвестициях в основной капитал занимают весьма существенный удельный вес - 42,7\%, в том числе прибыль предприятий в их сумме составляет примерно $17,2 \%$. Это почти сумма амортизации, предназначенная для воспроизводства основного капитала.

Прибыль не является гарантированным доходом предпринимателя, вложившего свой капитал в тот или иной вид бизнеса, это результат умелого и успешного осуществления бизнеса. Но в процессе ведения бизнеса предприниматель вследствие своих неудачных действий или объективных причин внешнего характера может не только лишиться ожидаемой прибыли, но и полностью или частично потерять вложенный капитал. Поэтому прибыль представляет в определенной мере и плату за риск осуществления предпринимательской деятельности.
В то же время необходимо видеть общеэкономическое значение прибыли предприятий в обеспечении финансовыми ресурсами устойчивого экономического роста.

Корреляционно-регрессионный анализ в рамках данной работы позволит провести оценку степени влияния на индекс инвестиций в основной капитал в Российской Федерации основных источников его формирования, которые в силу своей экономической природы определяют его величину и динамику.

Индекс инвестиций в основной капитал в Российской Федерации был выбран в качестве результативного признака $(Y)$ и заложен в основу исследования как самый информативный и наиболее полно отражающий развитие инвестиционной составляющей в стране. В качестве факторных признаков $(X)$, оказывающих влияние на динамику исследуемого показателя, отобраны следующие источники формирования:

$X_{1}$ - индекс фондов накопления в Российской Федерации, \% к предыдущему году; 
$X_{2}$ - индекс амортизации в Российской Федерации, \% к предыдущему году;

$X_{3}-$ индекс привлеченных кредитов банков, \% к предыдущему году.

Исходные данные для корреляционнорегрессионного анализа представлены в таблице 2.

Изучение взаимосвязи между явлениями начинается с установления ее тесноты, что производится методами корреляционного анализа. Основой измерения связей служит матрица парных коэффициентов корреляции. По этой матрице можно судить о тесноте связи факторов с результативным признаком и между собой. Парные коэффициенты корреляции рассчитываются следующим образом:

$$
r=\frac{\sum(x-\bar{x})(y-\bar{y})}{\sum(x-\bar{x})^{2}(y-\bar{y})^{2}},
$$

где $\bar{x}$ и $\bar{y}$ - средние значения результативного признака и соответствующего фактора.

С использованием ППП «Statistica» была получена матрица парных коэффициентов корреляции, используемая для анализа влияния на индекс инвестиций в основной капитал в Российской Федерации основных источников его формирования (табл. 3). Кроме полученных коэффициентов корреляции, в матрице указаны также вероятности принятия гипотезы об их незначимости $(p)$, установленные нами в рамках данного исследования в пределах 0,1 (или 10\%).

Величина рассчитанных коэффициентов корреляции позволяет судить о степени влияния факторных признаков на результативный показатель, а полярность («+» или «-») коэффициентов корреляции показывает тип влияния - прямое или обратное. Все рассчитанные коэффициенты корреляции имеют положительные значения, из которых следует, что наибольшее влияние на индекс инвестиций в основной капитал (Y) оказывают индексы фондов накопления $\left(\mathrm{X}_{1}\right)$ и привлеченных кредитов банков $\left(\mathrm{X}_{3}\right)$, т.е. с ростом значений этих индексов происходит рост индекса инвестиций в основной капитал. Заметное, но все же меньшее влияние на результативный признак оказывает индекс амортизации $\left(\mathrm{X}_{2}\right)$. Коэффициент его корреляции с результативным показателем имеет наименьшее значение $\left(\mathrm{r}_{\mathrm{YX} 2}=0,6117\right)$, его увеличение также способствует росту индекса инвестиций в основной капитал.

Таблица 2. Исходные данные для проведения корреляционно-регрессионного анализа степени влияния источников формирования на индекс инвестиций в основной капитал в РФ, \% к предыдущему году

\begin{tabular}{|c|c|c|c|c|}
\hline Год & $\begin{array}{c}\text { Индекс инвестиций } \\
\text { в основной капитал } \\
\text { в РФ }(Y)\end{array}$ & $\begin{array}{c}\text { Индекс фондов } \\
\text { накопления в РФ }\left(X_{1}\right)\end{array}$ & $\begin{array}{c}\text { Индекс амортизации } \\
\text { В РФ }\left(X_{2}\right)\end{array}$ & $\begin{array}{c}\text { Индекс привлеченных } \\
\text { кредитов банков }\left(X_{3}\right)\end{array}$ \\
\hline 2001 & 126,8 & 129,8 & 129,8 & 191,3 \\
\hline 2002 & 109,0 & 86,7 & 128,9 & 145,4 \\
\hline 2003 & 125,4 & 117,0 & 138,2 & 156,5 \\
\hline 2004 & 123,1 & 133,2 & 116,1 & 133,5 \\
\hline 2005 & 128,8 & 135,9 & 118,3 & 154,5 \\
\hline 2006 & 131,7 & 129,3 & 120,5 & 149,4 \\
\hline 2007 & 137,0 & 133,0 & 126,1 & 78,5 \\
\hline 2008 & 128,5 & 122,3 & 126,2 & 95,9 \\
\hline 2009 & 90,1 & 78,0 & 94,8 & 99,7 \\
\hline 2010 & 109,7 & 117,3 & 123,4 & \\
\hline 2011 & 116,2 & 117,5 & 122,4 & \\
\hline
\end{tabular}


Таблица 3. Матрица парных коэфффициентов корреляции

\begin{tabular}{|c|c|c|c|c|}
\hline & $Y$ & $X_{1}$ & $X_{2}$ & $X_{3}$ \\
\hline$Y$ & 1 & 0,8812 & 0,6117 & 0,7252 \\
& - & $p=0,000$ & $p=0,045$ & $p=0,012$ \\
\hline \multirow{2}{*}{$X_{1}$} & 0,8812 & 1 & 0,4076 & 0,5496 \\
& $p=0,000$ & - & $p=0,213$ & $p=0,080$ \\
\hline \multirow{2}{*}{$X_{2}$} & 0,6117 & 0,4076 & 1 & 0,5677 \\
& $p=0,045$ & $p=0,213$ & - & $p=0,069$ \\
\hline \multirow{2}{*}{$X_{3}$} & 0,7252 & 0,5496 & 0,5677 & 1 \\
\hline
\end{tabular}

Важным этапом проведения корреляционно-регрессионного анализа является отбор факторных признаков для последующего включения в уравнение множественной регрессии. Сложность формирования ее модели заключается в том, что факторные признаки нередко находятся в зависимости друг от друга, то есть являются мультиколлинеарными. На практике выявление случаев мультиколлинеарности осуществляется на основании матрицы парных коэффициентов корреляции. Индикатором ее наличия служит значение коэффициента корреляции $\mathrm{r}>0,8$.

Анализ матрицы парных коэффициентов корреляции (см. табл. 3) указывает на отсутствие мультиколлинеарности между факторными признаками $X_{1}, X_{2}$ и $X_{3}$, в связи с чем нет необходимости в исключении или замене какого-либо из них. Следовательно, в дальнейшую процедуру регрессионного анализа необходимо включить все три отобранных факторных признака. К тому же они оказывают существенное прямое влияние на величину результативного признака, и вероятность включения всех их в регрессионную модель, на наш взгляд, достаточно высока.

Перейдем к построению регрессионной модели. Данная математическая задача формулируется следующим образом: требуется найти аналитическое выражение зависимости экономического явления от определяющих его факторов, то есть необходимо найти функцию

$$
Y=f\left(X_{1}, X_{2}, \ldots, X_{\mathrm{k}}\right) \text {. }
$$

Цель состоит в том, чтобы раскрыть характер и степень влияния аргументов на функцию. Регрессионный анализ дает возможность установить, как в среднем изменяется зависимая величина с изменением одной или нескольких независимых величин при фиксированном значении неучтенных факторов. Кроме того, изучение парной зависимости между функцией и одним из аргументов обычно малоэффективно, поскольку экономические явления, как правило, многофакторны, а между факторами также существуют сложные взаимосвязи.

Процесс регрессионного анализа состоит из последовательности этапов:

- построение уравнения регрессии;

- проверка значимости коэффициентов уравнения регрессии;

- проверка значимости уравнения регрессии;

- расчет характеристик регрессии. 
В результате проведенного регрессионного анализа получено уравнение регрессии, которое имеет следующий вид:

$$
\begin{gathered}
Y=21,496+0,466 X_{1}+0,247 X_{2}+ \\
+0,103 X_{3},
\end{gathered}
$$

где $Y$ - оценочное значение результативного показателя.

Проверка значимости уравнения регрессии осуществляется с помощью F-критерия Фишера, расчетное значение которого находится по следующей формуле:

$$
F_{\text {расч. }}=\frac{D_{\text {общ. }}^{2}}{D_{\text {ост. }}^{2}}
$$

Общая дисперсия $D_{\text {общ. }}^{2}$ рассчитывается по следующей формуле:

$$
D_{\text {общ. }}^{2}=\frac{\sum(\bar{y}-y)^{2}}{k+1},
$$

где $k$ - число факторов;

$(k+1)$ - это число исследуемых факторов плюс свободный член в уравнении регрессии $a_{0}$;

$\bar{y}$ - среднее значение уровней результативного показателя.

Остаточная дисперсия $D$ определяется из выражения:

$$
D_{\text {ост. }}^{2}=\frac{\sum(\tilde{y}-y)^{2}}{n-k-1} \text {. }
$$

Расчетное значение F-критерия сравнивается с табличным, найденным при заданном уровне значимости $p$ и степенях свободы $(k+1)$ и $(n-k-1)$. Если расчетное значение критерия больше табличного, то гипотеза о незначимости построенного уравнения регрессии отвергается. Поэтому представляет интерес проверка значимости отдельных коэффициентов регрессии, осуществляемая с помощью t-критерия Стьюдента:

$$
t_{a_{j}}=\frac{a_{j}}{S_{a_{j}}},
$$

где $S_{a_{j}}$ - это среднее квадратическое отклонение коэффициентов уравнения $a_{i}$

$$
S_{a_{j}}=S_{e} \sqrt{b_{i j}}
$$

где $S_{\boldsymbol{B}}=\sqrt{\frac{\sum(y-\tilde{y})^{2}}{n-k-1}}-$ стандартное отклонение случайных ошибок;

$b_{i j}$ - диагональные элементы матрицы $\left(X^{T} X\right)^{-1}$.

Коэффициент регрессии признается значимым, если расчетное значение t-критерия Стьюдента больше табличного. Bce факторные признаки, коэффициенты регрессии при которых оказались незначимыми, исключаются из уравнения регрессии, процесс построения модели повторяется заново, т.е. с первого шага регрессионного анализа, и уравнение регрессии строится только на тех независимых переменных, коэффициенты при которых оказались значимыми. Затем

\begin{tabular}{|c|c|c|c|c|}
\hline \multicolumn{5}{|c|}{ Коэфффициент корреляции $R=0,9416$. Коэффрициент детерминации $R I=0,8867$} \\
\hline \multicolumn{5}{|c|}{ Значение критерия $F(3,7)=18,259, p<0,00109$} \\
\hline & $\beta$-коэфффициенты & параметры уравнения & $\begin{array}{c}\text { значение } \\
\mathrm{t}-\text { критерия Стьюдента (I) }\end{array}$ & уровень значимости $p$ \\
\hline Константа & & 21,496 & 1,056 & 0,326 \\
\hline$X_{1}$ & 0,664 & 0,466 & 4,317 & 0,003 \\
\hline$X_{2}$ & 0,202 & 0,247 & 1,292 & 0,238 \\
\hline$X_{3}$ & 0,246 & 0,103 & 1,441 & 0,093 \\
\hline
\end{tabular}
снова проверяется значимость уравнения регрессии и его коэффициентов. Так делается до тех пор, пока в уравнении не останутся только факторы со значимыми коэффициентами.

Таблица 4. Результаты регрессионного анализа на трех факторных признаках 
С вероятностью 0,90 полученные регрессионные коэффициенты многофакторной модели следует признать значимыми только у факторных признаков $X_{1}$ и $X_{3}$, так как вероятность принятия обратной гипотезы для них не превышает 0,1. При этом вероятность принятия обратной гипотезы для факторного признака $X_{2}$ (индекс роста амортизации) оказалась больше - 0,238 (табл. 4).

Исключим факторный признак $X_{2}$ из анализа и повторим процесс снова, т.е. построим уравнение регрессии только на тех независимых переменных, коэффициенты при которых признаны значимыми $\left(X_{1}\right.$ и $\left.X_{3}\right)$ (табл. 5).

В итоге получено уравнение регрессии, в которое вошли два значимых фактора:

$$
Y=43,785+0,485 X_{1}+0,144 X_{3} .
$$

F-критерий Фишера и t-критерий Стьюдента при уровне значимости $90 \%$ свидетельствуют о статистической значимости полученного уравнения и включенных в него факторных признаков.

Коэффициент детерминации, показывающий, какая часть общей вариации зависимой переменной определяется факторами, включенными в статистическую модель, рассчитывается по следующей формуле:

$$
R^{2}=\frac{\sum(\bar{y}-\tilde{y})^{2}}{\sum(y-\tilde{y})^{2}} \times 100 \%
$$

Коэффициент детерминации, равный 0,8597 , говорит о том, что на $85,97 \%$ динамика индекса инвестиций в основной капитал объясняется вошедшими в модель показателями $X_{1}$ и $X_{3}$, а уровень остаточной вариации составляет $14,03 \%$. Следовательно, на уровень инвестиций в основной капитал в Российской Федерации наибольшее влияние оказывают два источника формирования инвестиций - фонды накопления и объем привлеченных кредитов. Этот вывод подтверждает значение коэффициента множественной корреляции, равное 0,9271, свидетельствующее о высокой связи между результативным показателем и факторами, включенными в регрессионную модель.

Коэффициенты уравнения показывают абсолютный размер влияния факторов на уровень результативного показателя, характеризуют степень и тип влияния каждого фактора на анализируемый показатель. Их интерпретация позволяет сделать вывод о том, что индекс инвестиций в основной капитал ( $Y$ ) будет увеличиваться при увеличении индексов фондов накопления $\left(X_{1}\right)$ и привлеченных кредитов банков $\left(X_{3}\right)$. Согласно полученным данным, при повышении индекса фондов накопления на $1 \%$ индекс инвестиций в основной капитал увеличится на $0,485 \%$, а при росте индекса привлеченных кредитов банков на $1 \%$ индекс инвестиций в основной капитал возрастет на $0,144 \%$.

Таблица 5. Результаты регрессионного анализа на двух фракторных признаках

\begin{tabular}{|c|c|c|c|c|}
\hline \multicolumn{5}{|c|}{ Коэффрициент корреляции $R=0,9272$. Коэфрфициент детерминации $R I=0,8597$} \\
\hline & \multicolumn{4}{|c|}{ Значение критерия $F(2,8)=24,507, p<0,00039$} \\
\hline Конскоэффрициенты & $\begin{array}{c}\text { параметры } \\
\text { уравнения }\end{array}$ & $\begin{array}{c}\text { значение t-критерия } \\
\text { Стьюдента (I) }\end{array}$ & уровень значимости $p$ \\
\hline$X_{1}$ & 0,692 & 43,785 & 3,896 & 0,005 \\
\hline$X_{2}$ & 0,345 & 0,485 & 4,363 & 0,002 \\
\hline
\end{tabular}


Качество модели характеризует относительная ошибка аппроксимации $|\bar{\varepsilon}|$ рассчитываемая на основе разности между фактическими уровнями результативного признака $y$ и его расчетными уровнями $S$, т.е. уровнями, рассчитываемыми по регрессионной зависимости

$$
|\bar{\varepsilon}|=\frac{1}{n} \sum \frac{\mid y_{t}-\tilde{y}_{t}}{y_{t}} \times 100 \% .
$$

В нашем случае относительная ошибка в приближении для полученной модели составляет 3,64\% при норме 10-12\%.

Таким образом, можно достаточно обоснованно утверждать, что прибыль является важнейшим финансовым ресурсом инвестиционной активности современной организации. По отношению к валовому внутреннему продукту общая (валовая) прибыль предприятий составляет 12-15\%, а в наиболее благоприятные годы достигает почти 20\%. Убыточные предприятия не только снижают экономический и финансовый потенциал экономики, но и на деле лишены возможности проводить какуюлибо политику в области экономического развития, не говоря уже об инвестициях и инновациях. Удельный вес убыточных организаций в Российской Федерации (в процентах от их общего числа) в 2011 г. составил в целом по экономике $30,0 \%$. Следует отметить, что на таком высоком уровне он держится на протяжении всего периода реформирования отечественной экономики, хотя до этого считалось недопустимым его превышение 15-16\%.

Без кардинального решения данной проблемы говорить о повышении конкурентоспособности наших предприятий и создании благоприятных условий для инвестиционного и инновационного развития экономики не приходится. Убыточность не только нарушает воспроизводственный процесс конкретного предприятия, но и пагубно сказывается на воспроизводстве всего народного хозяйства, платежно-расчетных отношениях, что порождает целые цепочки аномалий в сложившейся экономической системе.

В то же время нужно иметь в виду, что удельный вес прибыльных организаций, по данным органов статистики, находится на весьма низком уровне.

В 2000-2011 гг. российская экономика имела весьма неустойчивые результаты финансовой деятельности как по сальдированной, так и по валовой прибыли. Ежегодные показатели в анализируемом периоде колебались от $30,9 \%$ спада до 75,7\% прироста сальдированной прибыли. Кроме того, даже у прибыльных предприятий наблюдалось весьма неустойчивое финансовое положение. Валовая прибыль в отдельные годы снижалась (в 2001 г. - на $15,6 \%$, в 2008 - на 16,5\%), а в 2010-2011 гг., на фазе выхода из экономического кризиса, наблюдалось ее увеличение в пределах 26 и $20 \%$ соответственно. Из этого можно сделать вывод, что инновационно-инвестиционная политика большинства российских предприятий не имела устойчивой ресурсной базы.

Отмечая важную роль прибыли в капитале корпоративного сектора, необходимо отметить происходящее в последнее время существенное уменьшение ее доли в общем объеме финансовых ресурсов. С одной стороны, число убыточных предприятий сокращается (если в 2003 г. их было 54 839, то в 2011 г. - 22 837), а с другой стороны, суммарная задолженность организаций по обязательствам на 2011 г. составила 1654030 млн. руб.

Несмотря на наметившуюся в российской экономике тенденцию к сокращению числа убыточных предприятий (организаций), сохранение низкого уровня рентабельности означает отсутствие налогооблагаемой базы для ряда налогов, прежде всего налога на прибыль. «Рационально 
разработанный налог на прибыль является налогом на отдачу от капитала, инвестированного в корпоративный сектор и, кроме того, налогом на дополнительную отдачу от инвестиций, скорректированную на риск. То есть налог на прибыль в действительности представляет собой предварительную (или частичную) часть налога на полный (совокупный) доход индивидуума» [4, c. 122].

Инвестиции, особенно в инновационную деятельность, по мере роста экономики диверсифицируются, становясь более эластичными к посленалоговой доходности. Становится невозможной дальнейшая эксплуатация правительством фиксированных инвестиций прошлых периодов с целью извлечения дохода в долгосрочном периоде. По этой же причине в развитых экономиках действует тенденция к снижению эффективных ставок налога на прибыль и оптимизации налогообложения.

Приведенный выше корреляционнорегрессионный анализ определяющего влияния валовой прибыли на инвестиционную активность предприятия подтверждает справедливость вывода о том, что налог на прибыль может вызвать нежелательные диспропорции в использовании факторов производства (труда и капитала), порождая неэффективную комбинацию этих факторов, тем самым снижая стимулы к инновационно-инвестиционной деятельности.

В сложившейся ситуации конкретная задача эффективной налоговой политики состоит в том, чтобы формирование налоговой ставки на прибыль осуществлялось в максимально стимулирующей форме с учетом эффекта Лаффера. Кроме того, необходимо обеспечить соответствие уровней номинального и фактического налогового бремени предприятий. Пока не ясно, на каком уровне от ВВП или добавленной стоимости такое соответствие может быть достигнуто. Вместе с тем можно назвать диапазон возможных значений номинального налогового бремени (по действующим налогам), который, как показывает опыт зарубежных стран, колеблется в пределах 30-40\% от ВВП. Причем если при уровне налогообложения $30 \%$ от ВВП (согласно опыту США) могут быть обеспечены более быстрое развитие экономики и рост налоговых поступлений в будущем, то при $40 \%$ можно иметь большие текущие поступления в бюджет при меньших темпах развития экономики.

Налоговый механизм, обеспечивающий формирование благоприятного экономического климата для развития инвестиционной деятельности, должен соответствуюшим образом взаимодействовать с кредитно-банковской политикой, процентными ставками, предоставляя определенные преимущества предприятиям, использующим долгосрочные ссуды на инвестиционные цели.

Логика экономического развития такова, что ставки по кредитам в целом должны быть ниже уровня средней прибыли в экономике. Только при этих условиях у предпринимателей появится возможность пользоваться заемными средствами. В свою очередь, кредитные ставки коммерческих банков должны быть выше ставки рефинансирования и ставок по вкладам, иначе банки просто не смогут эффективно работать. И наконец, все участники кредитного рынка должны иметь реальный процентный доход, т.е. уровень номинальных процентных ставок должен быть выше инфляции.

В российской экономике на современном этапе можно наблюдать достаточно неблагоприятную картину: средний уровень рентабельности бизнеса сравнительно ниже уровня средневзвешенных ставок по кредитам и финансовым организациям. 
Такое соотношение между рентабельностью реального сектора российской экономики и ставкой по кредитам объясняет многие существующие на сегодняшний момент проблемы отечественной экономики, связанные с недостаточным инвестированием и уходом капитала в спекулятивные операции, а также его оттоком за границу. Итак, чем выше эффективность инвестиционных проектов и чем ниже банковский процент, тем интенсивнее инвестиционная деятельность и предпринимательская активность. Чем дороже кредитные ресурсы, тем меньше в экономике эффективных бизнес-проектов, тем меньше на них спрос инвесторов.

Высокие процентные ставки по ссудам препятствуют перемещению кредитных ресурсов в реальный сектор и обусловливают его технологическую деградацию, что делает российский рынок малопривлекательным для инвесторов. Для российских предпринимателей оказывается выгоднее вкладывать капиталы за границу, где реальный сектор дает возможность получать прибыль, пользуясь кредитными ресурсами по умеренным ставкам.

В последние десятилетия в мировой практике используется ряд специфических финансовых инструментов, направленных на участие государства в разделении кредитных и инвестиционных рисков при осушествлении инвестиционных проектов. Нередко применяются такие механизмы, как предоставление предприятиям государственных гарантий для получения долгосрочных займов от частных кредитных учреждений, долевое акционерное участие государства в формировании фондов венчурного капитала и инвестиционном бизнесе, защита национальных производителей от иностранных конкурентов и др.

Гарантированные кредиты дают возможность поддерживать наукоемкие про- екты, связанные с высокими или трудно поддающимися оценке кредитными рисками. Существующая практика предусматривает участие в инвестиционном процессе третьей стороны (гаранта), берущей на себя всю полноту ответственности за выполнение обязательств заемщика перед кредитором. Таким образом, заемщики могут брать кредиты, на получение которых в иных условиях практически нельзя было бы рассчитывать.

Другая распространенная форма снижения инвестиционных рисков - долевое акционерное участие государства в тех или иных проектах на правах партнера с ограниченной ответственностью.

Механизмы государственных гарантий способствуют усилению частно-государственного партнерства благодаря разделению рисков между частными инвесторами и уполномоченными государственными организациями, осуществляющими предоставление таких гарантий. В результате не только увеличивается приток частного капитала, но и повышается эффективность использования ограниченных бюджетных ресурсов.

Акционерное финансирование позволяет аккумулировать крупные финансовые ресурсы путем размещения акций среди неограниченного круга инвесторов (заем денег у покупателей акций на неопределенное время) для осуществления перспективных инновационных проектов. Посредством эмиссии ценных бумаг производится замена инвестиционного кредита рыночными долговыми обязательствами, что способствует оптимизации структуры финансовых ресурсов, инвестируемых в инновационный проект.

Понятно, что ни один инвестор не желает рисковать в одиночку, ему будет спокойнее, если его деятельность будет поддерживаться государственной властью. 
Хорошей страховкой риска, по нашему мнению, станет совместное участие в проекте государственного и частного капиталов, которые одинаково заинтересованы в том, чтобы не только вернуть свои деньги, но и получить прибыль.

Усилия по стимулированию инвестиционной деятельности должны быть сконцентрированы на устранении основных причин незаинтересованности бизнеса в долгосрочных проектах. Среди наиболее важных проблем, которые требуют первоочередной разработки механизмов государственного регулирования инвестиционного процесса, можно назвать следующие:

- поддержка и развитие новых форм кредитования инвестиционных проектов в Российской Федерации;

- предложения об условиях предоставления государственных гарантий в отношении привлеченных в инвестиционную сферу кредитов;

- развитие механизмов консолидации финансовых ресурсов государственного и частнопредпринимательского секторов экономики для реализации приоритетных научно-технических задач, содействие формированию и регулирование деятельности партнерств частного сектора и государства;

- разработка механизмов оценки государственных инвестиционных программ и доведения результатов этой оценки до делового и экспертного сообщества;
- содействие формированию международных и региональных технологических стратегических альянсов.

Становится все более очевидным тот факт, что обеспечение высоких и устойчивых темпов развития России невозможно без взаимовыгодного партнерства государственных муниципальных органов власти, коммерческих банков и представителей бизнеса.

Экономические стратегии и программы, ориентированные только на использование бюджетных средств и базирующиеся на ортодоксальной неоклассичекой доктрине, недостаточны для развития инновационной экономики, лежащей в основе высокой конкурентоспособности страны.

Взаимодействие государства и бизнеса при финансировании инвестиционного процесса должно базироваться на развитии паритетно-партнерских отношений, которые предполагают взаимовыгодное сотрудничество и обеспечивают необходимые условия для «формирования качественно нового государства, способного выполнить план национального развития и решить многие, в том числе непростые геополитические, задачи» $[5$, c. 6$]$.

В заключение необходимо отметить, что только реализация комплекса предложенных мер будет способствовать активизации инвестиционного процесса в Российской Федерации.

\section{Литература}

1. Ильин, В. А. Проблемы эффективности государственного управления. Бюджетный кризис регионов / В. А. Ильин, А. И. Поварова. - Вологда: ИСЭРТ РАН, 2013. - 128 с.

2. Кормишкина, Л. А. Деформации общественного воспроизводства: причины, анализ, противодействие [Текст] / Л. А. Кормишкина, Д. А. Колосков. - Саранск: [б. и.], 2011. - 144 с.

3. Российский статистический ежегодник / Росстат. - М., 2012. - 786 с.

4. Проблемы налоговой системы России: теория, опыт, реформа [Текст] / под ред. М. Алексеева, С. Синельникова: в 2 т. - М.: Ин-т экономики переход. периода, 2000. - Т. 1. - 606 с.

5. Федоров, Е. А. За частным бизнесом - приоритет в государственном партнерстве [Текст] / Е. А. Федоров // Государственно-частное партнерство. Пути совершенствования законодательной базы / под общ. ред. А. А. Зверева. - М., 2009. - 238 с. 
Kormishkina L.A., Yulenkova I.B.

\section{Investment process financing in Russian business: assessment, trends, problems}

Lyudmila Aleksandrovna Kormishkina - Doctor of Economics, Professor, Federal State-Financed Educational Institution of Higher Professional Education "Ogarev Mordova State University" (68, Bolshevistskaya Street, Saransk, Republic of Mordovia, 430005, Russia, larisa-mist@rambler.ru)

Irina Borisovna Yulenkova- Ph.D. in Economics, Associate Professor, Federal State-Financed Educational Institution of Higher Professional Education "Ogarev Mordova State University" (68, Bolshevistskaya Street, Saransk, Republic of Mordovia, 430005, Russia, larisa-mist@rambler.ru)

Abstract. In modern conditions the provision of investment process financing is the most important task of state economic policy aimed at achieving sustainable growth and dynamic development of the Russian economy in general. This problem solution requires further theoretical consideration and development of appropriate methodological, methodical and practical recommendations. Financing of investment activity development should be based on the systemic approach, which considers this process as an element of the financial support system of the state innovation development. It is necessary to conduct research in order to expand the financial component of this support, encourage the investment process development, enhance financial relations in the sphere of forming and using the intellectual property objects and develop a complex of measures to study the innovations implementation possibilities. Although economic science pays a lot of attention to various aspects of the issue, there is currently no research work, devoted to the study of sources of the investment process financing. Development of methodical and practical recommendations to establish the system of its financing, taking into account modern world economic trends is required. The authors have revealed the enterprises' general economic profit while promoting sustainable economic growth by means of provision of financial resources for it. The closeness of the relationship is calculated on the basis of regression models that characterize the sectoral distribution of gross profit. The correlation-regression analysis has helped to assess the influence of the main sources of the RF fixed assets on the investment index. The economic nature of the sources determines its value and dynamics.

Key words: investment process, investment climate, business, intensification, profit, source of financing, investment costs, regression model, indices, dynamics, investment operations, investment activity, the level of taxation.

\section{References}

1. Ilyin V.A., Povarova A.I. Problemy effektivnosti gosudarstvennogo upravleniya. Byudzhetnyi krizis regionov [Public Administration Efficiency. The Fiscal Crisis of the Regions]. Vologda: ISERT RAN, 2013. 128 p.

2. Kormishkina L.A., Koloskov D.A. Deformatsii obshchestvennogo vosproizvodstva: prichiny, analiz, protivodeistvie [Deformation of Social Reproduction: Causes, Analysis, Counteraction]. Saransk, 2011. 144 p.

3. Rossiiskii statisticheskii ezhegodnik [Russian Statistical Yearbook]. Rosstat, Moscow, 2012. 786 p.

4. Problemy nalogovoi sistemy Rossii: teoriya, opyt, reforma [Problems of the Russian Tax System: Theory, Experience, Reform]. Ed. by M. Alekseev, S. Sinel'nikova: in 2 volumes. Moscow: In-t ekonomiki perekhod. perioda, 2000. Vol. 1. 606 p.

5. Fedorov E.A. Za chastnym biznesom - prioritet $\mathrm{v}$ gosudarstvennom partnerstve [Private Business is a Priority of State Partnership]. Gosudarstvenno-chastnoe partnerstvo. Puti sovershenstvovaniya zakonodatel'noi bazy [PublicPrivate Partnership. Ways of Legislative Base Betterment]. Under general editorship of A.A. Zverev. Moscow, 2009. $238 \mathrm{p}$. 